\title{
Active silicon octagonal micropillar resonator modulators using metal-oxide-semiconductor capacitors
}

\author{
Chao Li and Andrew W. Poon \\ Department of Electrical and Electronic Engineering, The Hong Kong University of Science and Technology, \\ Clear Water Bay, Hong Kong SAR, China \\ Tel: (852)-2358-7905, fax: (852)-2358-1485, email: eeawpoon@ust.hk
}

\begin{abstract}
We propose active silicon waveguide-coupled octagonal micropillar resonator modulators using metal-oxide-semiconductor capacitors. Our initial electrical and optical simulations suggest a high-extinction ratio resonance modulation by selectively embedding capacitors along the octagonal micropillar rim region.

(C) 2005 Optical Society of America

OCIS codes: (230.5750) Resonators; (230.4110) Modulators
\end{abstract}

\section{Summary}

Compact high-speed silicon electro-optical modulators with low-power consumption are one of the building blocks for emerging silicon photonic chips. Recently, silicon-on-insulator (SOI) GHz-bandwidth electro-optical modulators have been experimentally demonstrated using metal-oxide-semiconductor (MOS) capacitor-based phase shifters integrated on Mach-Zehnder interferometers [1]. This pioneering work, however, has the shortcoming of imposing relatively long mm-cm length phase shifters, and thus a relatively large power consumption. In order to attain compact-size phase-sensitive tunable silicon components, Barrios et al. [2] proposed and demonstrated SOI Fabry-Perot microresonators that are tuned by means of carrier injection from conventional lateral p-i-n diodes. In our previous work [3], we proposed and analyzed compact SOI waveguide-coupled microring resonator modulators using MOS capacitors. Although our simulations suggested GHz-bandwidth resonance modulation, practical concerns include (i) the short coupling length between the circular microring and the side-coupled waveguide, (ii) the microring inner-sidewall scattering loss, and (iii) opening contact holes on the submicrometer width MOS capacitor ring.

(a)

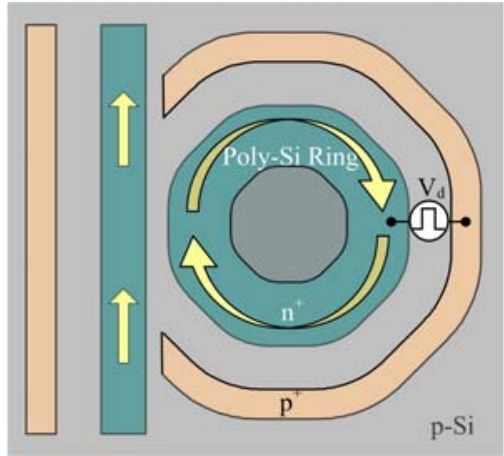

(b)

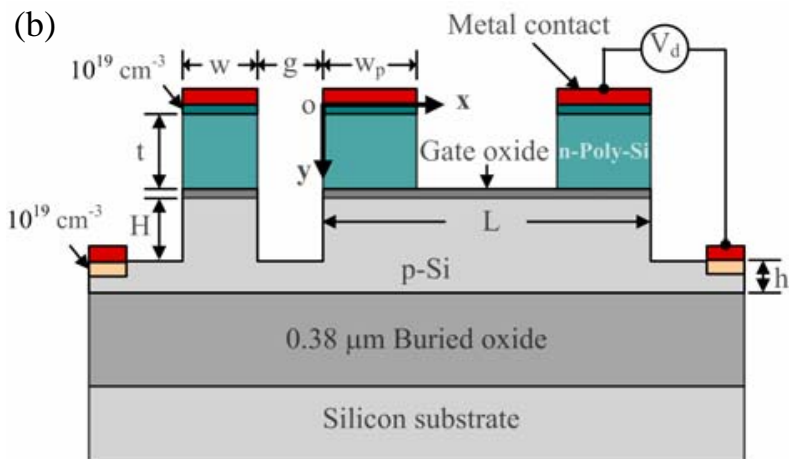

Fig. 1. (a) Schematic of an SOI waveguide-coupled round-corner octagonal $\mu$-pillar resonator modulator using MOS capacitors. The arrows represent the lightwave propagating inside the waveguide and the microresonator. $\mathrm{V}_{\mathrm{d}}$ is the driving voltage. (b) Schematic cross section. $\mathrm{L}=3 \mu \mathrm{m}, \mathrm{w}=0.3 \mu \mathrm{m}, \mathrm{g}=0.2 \mu \mathrm{m}, \mathrm{w}_{\mathrm{p}}=0.5 \mu \mathrm{m}, t=0.2 \mu \mathrm{m}, H=0.24$ $\mu \mathrm{m}, h=0.1 \mu \mathrm{m}$. The entire device is cladded by LTO.

In this summary we propose alternative active SOI waveguide-coupled round-corner octagonal micropillar ( $\mu$-pillar) resonator modulators using MOS capacitors. We outline an analysis that includes both electrical and optical simulations. Our initial simulations suggest a high-extinction ratio resonance modulation by selectively embedding MOS capacitors along the octagonal $\mu$-pillar rim region. 
Waveguide-coupled round-corner octagonal $\mu$-pillar resonators have the major advantage of long lateral coupling length along the entire flat cavity sidewalls, and thus potentially ease the tight fabrication constraint of the submicrometer air-gap separation between the microresonator and the side-coupled waveguide. The round-corner design helps mitigate the octagonal $\mu$-pillar resonator loss, and enables whispering-gallery (WG) like modes [4]. Multiple WG-like orbits of the same mode can be coherently waveguide-coupled along the entire flat cavity sidewall [4].

Figures 1 (a) and 1 (b) show the top-view and cross-sectional schematics of an SOI waveguide-coupled round-corner octagonal $\mu$-pillar resonator modulator using MOS capacitors. We selectively integrate the MOS capacitor structure along the $\mu$-pillar rim region (and the coupled waveguide) in order to selectively tune the refractive index of the WG-like modes. The MOS capacitor is comprised of an n-doped polysilicon top layer (with about $10^{19} \mathrm{~cm}^{-3}$ surface doping), a nanometer-sized oxide layer, and a p-doped silicon bottom layer. The silicon slab layer adjacent to the MOS structure is $\mathrm{p}^{+}$doped (with about $10^{19} \mathrm{~cm}^{-3}$ surface doping). The $\mathrm{p}^{+}$doped ring surrounds most of the $\mu$-pillar except the coupling sidewall. The polysilicon ring width can be sufficiently wide for the ease of opening contact holes. The entire device is cladded by low-temperature oxide (LTO).
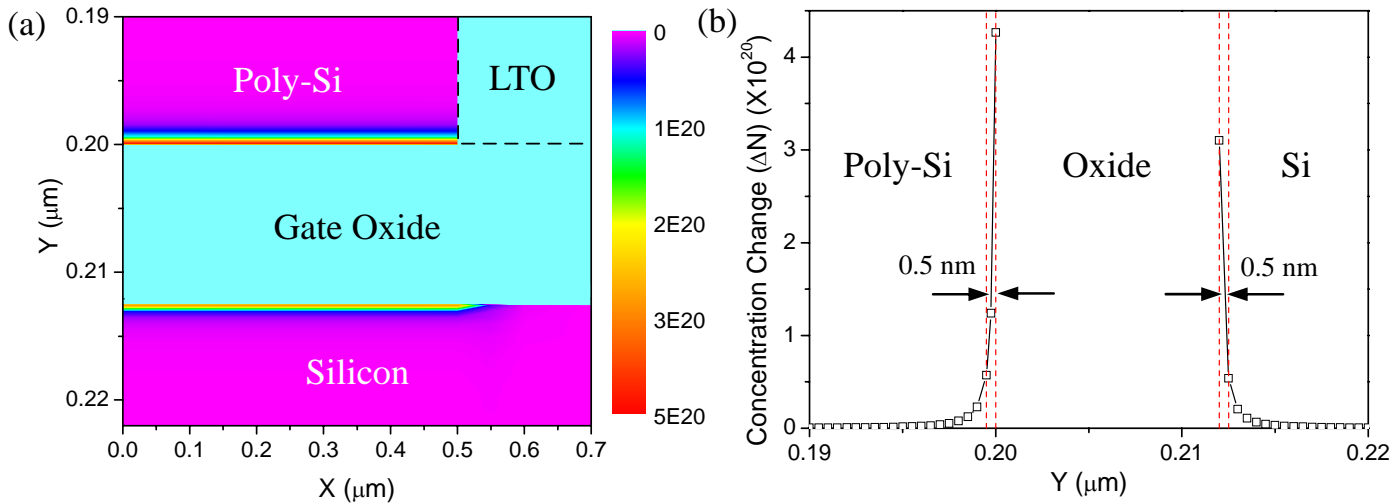

Fig. 2. (a) Contour of the free-carrier concentration change on both sides of the gate oxide. (b) Profile of the free-carrier concentration change at $\mathrm{x}=0.25 \mu \mathrm{m}$.

We employed a two-dimensional (2-D) device simulator MEDICI to numerically study the device electrical characteristics. Figure 2 (a) shows the calculated contour of the free-carrier concentration change in the p-doped silicon (hole carrier concentration change $\Delta \mathrm{N}_{\mathrm{h}}$ ) and the n-doped polysilicon (electron carrier concentration change $\Delta \mathrm{N}_{\mathrm{e}}$ ) region on both sides of the $12 \mathrm{~nm}$ gate oxide at the $\mu$-pillar rim region (Fig. 1 (b)), upon a relatively large driving voltage of $9 \mathrm{~V}$. The contour suggests that the $\Delta \mathrm{N}_{\mathrm{e}, \mathrm{h}}$ in the $\mathrm{x}$-direction is uniform (away from the vicinity of the polysilicon sidewall). Figure 2 (b) shows the $\Delta \mathrm{N}_{\mathrm{e}, \mathrm{h}}$ profile (non-exponential) in the y-direction at $\mathrm{x}=0.25 \mu \mathrm{m}$. The free-carriers only effectively accumulate on a thin 0.5-nm thick layer on both sides of the gate oxide. For further analysis, we approximate each of the two charge layers as an 0.5-nm thin charge layer, with an effective concentration change $\Delta \mathrm{N}_{\mathrm{e} \text {,eff }}$ and $\Delta \mathrm{N}_{\mathrm{h} \text {,eff }}$ defined as the integrated concentration change over the entire accumulation layer normalized by $0.5 \mathrm{~nm}$.

Applying the well-known relations for free-carrier plasma dispersion effect in bulk materials [5], we approximately calculate the $\Delta \mathrm{N}_{\mathrm{e} \text {,eff }}$ and $\Delta \mathrm{N}_{\mathrm{h} \text {,eff }}$ induced variation in real refractive index $\Delta \mathrm{n}$ and in absorption coefficient $\Delta \alpha$ at $1.55 \mu \mathrm{m}$ wavelengths for the effective subnanometer-thin surface charge layers. The approximated $|\Delta \mathrm{n}|$ and $\Delta \alpha$ are about 0.4 and $3700 \mathrm{~cm}^{-1}$ at the polysilicon-oxide interface, and is about 0.24 and 1900 $\mathrm{cm}^{-1}$ at the silicon-oxide interface.

On the optical simulations front, we first employed the full-vector beam propagation method (BPM) in order to calculate the change in effective refractive index $\Delta \mathrm{n}_{\text {eff }}$ and in effective absorption coefficient $\Delta \alpha_{\text {eff }}$ for the waveguide mode, assuming the approximated $\Delta \mathrm{n}$ and $\Delta \alpha$ at the effective subnanometer-thin surface charge layers. 
(a)

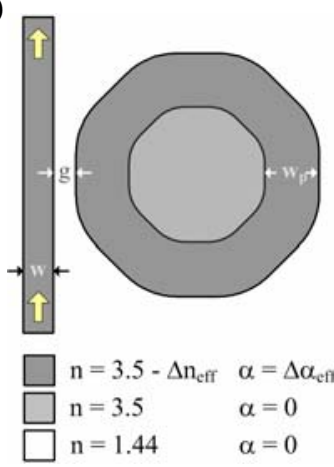

(b)

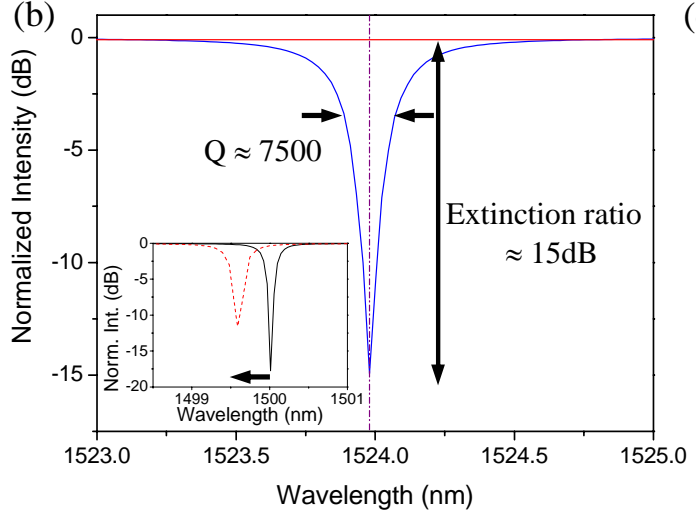

(c)

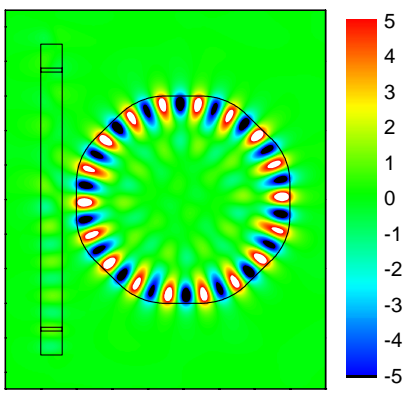

Fig. 3. (a) FDTD simulation schematic of an active SOI waveguide-coupled round-corner octagonal $\mu$-pillar resonator. (b) FDTD simulated resonance modulation from the off-state (solid line) to the on-state (dashed line). Inset shows the resonance modulation for a waveguide-coupled circular microdisk with the same design parameters. (c) Traveling-wave WGM field pattern for the off-state.

We then adopted the $\Delta \mathrm{n}_{\text {eff }}$ and $\Delta \alpha_{\text {eff }}$ for the two-dimensional finite-difference time-domain (FDTD) simulations in order to model the resonance modulation response. Figure 3 (a) shows the FDTD-simulation schematic of an active waveguide-coupled round-corner octagonal microresonator with MOS capacitors. The dark gray regions indicate the MOS embedded region where $\Delta \mathrm{n}_{\text {eff }}$ and $\Delta \alpha_{\text {eff }}$ are assumed when a forward bias $(9 \mathrm{~V})$ is applied. In order to simulate the refractive index contrast between silicon and LTO, both the waveguide and the $\mu$-pillar assume a refractive index $n=3.5$ while the background refractive index is 1.44 . Figure 3 (b) shows the FDTD simulated resonance modulation by assuming the same $\Delta \mathrm{n}_{\text {eff }}=-0.001$ and $\Delta \alpha_{\text {eff }}=7.2 \mathrm{~cm}^{-1}$ at the rim region and the input-coupled waveguide. When no voltage is applied, the FDTD-calculated resonance (blue line) has a $\mathrm{Q} \approx 7,500$ and a coupling efficiency of about $97 \%$. Figure 3 (c) shows the traveling-wave WGM field pattern for the off-state. When a $9 \mathrm{~V}$ forward bias is applied, contrary to our expectation based on our previous active microring work [3], the FDTD-calculated resonance (red line) vanishes. This unexpected result suggests that an extinction ratio exceeding $15 \mathrm{~dB}$ can be attained at the resonance wavelength. In order to verify our analysis, we simulated a waveguide-coupled circular microdisk with the same design parameters. Inset shows the resonance blueshift with linewidth broadening, as expected from our previous active microring work [3]. Our unexpected simulation results thus hint to a resonance coupling mechanism for waveguide-coupled octagonal $\mu$-pillar that is particularly sensitive to a small change in refractive index and absorption.

In summary, we report an initial analysis of active SOI waveguide-coupled round-corner octagonal $\mu$-pillar resonator modulators using MOS capacitors. By selectively embedding the MOS capacitors along the octagonal $\mu$-pillar rim region, we obtained a resonance modulation with an extinction ratio of $15 \mathrm{~dB}$. Further design parameter optimization and device fabrication on thin-film SOI substrates using standard CMOS processes are in progress.

\section{References}

[1] A. Liu, R. Jones, L. Liao, D. Samara-Rubio, D. Rubin, O. Cohen, R. Nicolaescu and M. Paniccia, “A high-speed silicon optical modulator based on a metal-oxide-semiconductor capacitor”, Nature, vol. 427, pp. 615 - 618, 2004.

[2] C. A. Barrios, V. R. Almeida, R. R. Panepucci, B. S. Schmidt, M. Lipson, “Compact silicon tunable Fabry-Perot resonator with low power consumption,” IEEE Photonics Tech. Lett., vol. 16, pp. 506 -508, 2004.

[3] C. Li and A. W. Poon, “Active silicon microring resonators using metal-oxide-semiconductor capacitors,” in proceedings of IEEE/LEOS 1st international Conference on Group IV Photonics, Hong Kong S.A.R., China, Sep. 29 - Oct. 1, 2004.

[4] C. Li and A. W. Poon, "Experimental demonstration of waveguide-coupled round-corner octagonal microresonators in silicon nitride", Opt. Lett., to be published.

[5] R. A. Soref and B. R. Bennett, “Kramers-Kronig analysis of electro-optical switching in silicon”, in Proc. SPIE, vol. 704, pp. 32-37, 1986.. 